\title{
A spatial and spectral maximum entropy method as applied to OVRO solar data
}

\author{
R.W. Komm, G.J. Hurford, and D.E. Gary \\ Solar Astronomy 264-33, Caltech, Pasadena, CA 91125, U.S.A.
}

Received September 23, 1995; accepted June 8, 1996

\begin{abstract}
We present first results of applying a Maximum Entropy Method (MEM) algorithm that acts in both the spatial and spectral domains to data obtained with the frequency-agile solar interferometer at Owens Valley Radio Observatory (OVRO) taken at 45 frequencies in the range $1-18 \mathrm{GHz}$. The traditional MEM algorithm does not exploit the spatial information available at adjacent frequencies in the OVRO data, but rather applies separately to each frequency. We seek an algorithm that obtains a global solution to the visibilities in both the spatial and spectral domains. To simplify the development process, the algorithm is at present limited to the onedimensional spatial case. We apply our 1-d algorithm to observations taken with the OVRO frequency-agile interferometer of active region AR 5417 near the solar limb on March 20, 1989 (vernal equinox). The interferometer's two $27 \mathrm{~m}$ antennas and $40 \mathrm{~m}$ antenna were arranged in a linear east-west array, which at the vernal equinox gives a good match to the 1-d algorithm. Our results show that including the spectral MEM term greatly improves the dynamic range of the reconstructed image compared with a reconstruction without using this information. The derived brightness temperature spectra show that for AR 5417 the dominant radio emission mechanism is thermal gyroresonance and we use this information to deduce the spatial variation of electron temperature and magnetic field strength in the corona above the active region.
\end{abstract}

Key words: Sun: corona - Sun: magnetic fields Sun: radio radiation - techniques: image processing techniques: interferometric

\section{Introduction}

In this paper, we present a Maximum Entropy Method (MEM) algorithm that acts in both the spatial and spectral domains and we show first results of applying this

Send offprint requests to: R. Komm algorithm to data obtained with the frequency-agile solar interferometer at Owens Valley Radio Observatory (OVRO). The data are taken at 45 frequencies in the range $1-18 \mathrm{GHz}$ and measure the Fourier components of the brightness distribution, where each frequency measures a different spatial component. The measured Fourier data are used to reconstruct an image in the spatial domain, as a function of position and frequency, to provide brightness temperature spectra at each point which can be analyzed and interpreted in terms of physical parameters such as coronal magnetic field strength and electron temperature. The number of observed Fourier components can be quite large depending on the array and the length of the observing time, but not all Fourier components will be measured which can lead to ambiguities in interpreting the data. Thus, one has to address the problem of missing information or incomplete $(u v)$ coverage. Imaging algorithms, such as CLEAN or the standard MEM algorithm, fill in the unmeasured Fourier components by using a priori information about what the radio source is expected to look like. However, the existing algorithms do not exploit the spatial information available at adjacent frequencies in the OVRO data. These algorithms treat each frequency separately which leads to a reconstructed image consisting of 45 independent spatial maps. We present an algorithm that obtains a global solution to the visibilities in both the spatial and spectral domains.

The spatial term in the traditional least-squares MEM maximizes a spatial entropy term $S_{\mathrm{spa}}=-\sum T \ln T$, where $T$ is the map temperature as a function of spatial position and frequency, subject to the data constraints. This ensures that the resulting image is spatially smooth and that it is positive everywhere. In our modified algorithm, we include an analogous spectral entropy term defined as $S_{\text {spe }}=-\sum \tau \ln \tau$, with $\tau=1+\left|T-T^{\prime}\right|$ where $T^{\prime}$ is the temperature interpolated from the two neighboring frequencies at the same spatial position. This term ensures that a spectrum at a given spatial position changes only smoothly with frequency. In this paper, the algorithm is described in one spectral and two spatial dimensions, but is applied only for the case of one spatial and one spectral 
dimension for several reasons: (1) To simplify the development process; (2) To speed up exploration of the relevant parameter space; (3) To make presentation of the results simpler (we display the one spectral and one spatial dimension as 2-d contour plots, whereas two spatial and one spectral dimensions would require presentation of a datacube). A subsequent paper is planned to describe the full algorithm as applied to one spectral and two spatial dimensions.

In Sect. 2, we describe the concept and its current implementation. In Sect. 3, we apply our 1-d algorithm to observations taken with the OVRO frequency-agile interferometer of active region AR 5417 near the solar limb on March 20, 1989 (vernal equinox) using the two 27-m antennas and the 40-m antenna arranged in a linear eastwest array. The geometry of an east-west array on this date gives strictly 1-d spatial resolution, and so gives a good match to the 1-d algorithm. We compare the result of our MEM algorithm with the source structure shown on 1-d OVRO maps obtained using the conventional CLEAN algorithm, and with a reconstruction using only the spatial MEM term of our algorithm. Then, we use the reconstructed image to calculate brightness temperature spectra and to derive physical parameters.

\section{The spatial and spectral MEM algorithm}

\subsection{The concept}

Although the data used in subsequent sections were obtained with a linear array of three antennas as noted above, our ultimate goal for the algorithm is to apply it to data from the 5-element solar interferometer at Owens Valley Radio Observatory (OVRO). This array currently consists of two 27-m antennas and three 2-m antennas with frequency-agile receivers, and is capable of generating 2-dimensional maps of solar sources at 45 frequencies in the range $1-18 \mathrm{GHz}$. See Gary \& Hurford (1994) for an example of observations with the complete five-element array. The algorithm is thus being developed to handle fully three-dimensional data (one spectral and two spatial dimensions). Since this is the more general case, in this subsection we conceptually describe the complete 3 -d algorithm. We implement and apply it to the simpler case of one spatial dimension in subsequent sections.

A typical interferometer array measures the spatial Fourier components of the brightness distribution at each frequency, $\nu$, and the observations consist of data pairs of flux amplitude, $A_{\nu, b}$, and phase, $\phi_{\nu, b}$, as a function of frequency, $\nu$, and baseline, $b$. Each data pair represents one point in a complex visibility plane:

$A_{\nu, b} \mathrm{e}^{i \phi_{\nu, b}} \equiv V_{\nu}(u, v)$.

The image of the observed radio source or its "true" map, $T(x, y, \nu)$, as a function of spatial position and fre- quency has to be positive everywhere (continuum emission), and is related to the data via a Fourier transform.

$$
\begin{aligned}
V_{\nu}(u, v) & =k \nu^{2} \iint T(x, y, \nu) \mathrm{e}^{i 2 \pi u x} \mathrm{e}^{i 2 \pi v y} \mathrm{~d} x \mathrm{~d} y+\text { noise } \\
& \equiv X_{\nu, b}+i Y_{\nu, b}
\end{aligned}
$$

with

$$
\begin{aligned}
X_{\nu, b}=k \nu^{2} \int & \int T(x, y, \nu)[\cos 2 \pi u x \cos 2 \pi v y \\
& -\sin 2 \pi u x \sin 2 \pi v y] \mathrm{d} x \mathrm{~d} y+\text { noise } \\
Y_{\nu, b}=k \nu^{2} \int & \int T(x, y, \nu)[\cos 2 \pi u x \sin 2 \pi v y \\
& +\sin 2 \pi u x \cos 2 \pi v y] \mathrm{d} x \mathrm{~d} y+\text { noise }
\end{aligned}
$$

where $k$ is the Boltzmann constant, $x$ and $y$ are the spatial coordinates and $u=\nu b_{x} / c$ and $v=\nu b_{y} / c$ are the corresponding spatial frequencies, which are functions of observing frequency, $\nu$, and projected baseline lengths, $b_{x}$ and $b_{y}$. For spatially 1 -d data $(v=0)$, this relation reduces to

$X_{\nu, b}=k \nu^{2} \int T(x, y, \nu) \cos 2 \pi u x \mathrm{~d} x+$ noise,

$Y_{\nu, b}=k \nu^{2} \int T(x, y, \nu) \sin 2 \pi u x \mathrm{~d} x+$ noise.

The measurement consists of a finite number of generally unevenly spaced points in the Fourier domain. To reconstruct an image of the radio source, unmeasured Fourier components have to be taken into account. If they are simply set to zero, the resulting "dirty map" will show strong sidelobes, which can make it difficult to interpret the image. Thus, our objective is to approximate the true image with more likely values than zero for the unmeasured components, but without violating the data constraints; a general discussion of the imaging problem for radio data can be found in Christiansen \& Hogbom (1985) and Cornwell \& Braun (1989). Since the antennas of the OVRO solar array are equipped with frequency-agile receivers and existing algorithms such as CLEAN (Högbom 1974) or standard MEM do not take advantage of the spatial information available at adjacent frequencies, we wish to develop an algorithm that obtains a global solution to the visibilities in both the spatial and spectral domains.

For this task, following the usual procedure for a MEM algorithm, we chose a maximum entropy method which allows us to reconstruct a temperature map consistent with the data with the least amount of added a priori information or with the fewest assumptions. Narayan \& Nityananda (1986) give a good description of MEM and its application to radio data, for a more general description of MEM see, for example, Bevensee (1993). The standard least-squares MEM ensures that the resulting image 
is spatially smooth and that it is positive everywhere. We add to it the information or assumption that a spectrum at a given spatial position changes only smoothly with frequency. Thus, our goal can be defined as finding the temperature map, $T^{(n)}(x, y, \nu)$, which maximizes the following objective function:

$r^{(n)}=\lambda S_{\mathrm{spa}}^{(n)}+\psi S_{\mathrm{spe}}^{(n)}-\Delta^{(n)}$,

where $S_{\text {spa }}^{(n)}$ and $S_{\text {spe }}^{(n)}$ are the spatial and the spectral entropy terms, $\Delta^{(n)}$ is the data constraint, $\lambda$ and $\psi$ are two (adjustable) Lagrange multipliers and $n$ is the iteration index.

As in standard MEM, the data constraint is expressed as a least-squares term

$\Delta^{(n)}=\frac{1}{N_{\nu}} \frac{1}{N_{b}} \sum_{\nu, b} \frac{1}{\eta_{\nu, b}^{2}}\left[\left(X_{\nu, b}^{(n)}-X_{\nu, b}\right)^{2}+\left(Y_{\nu, b}^{(n)}-Y_{\nu, b}\right)^{2}\right](8)$

where $X_{\nu, b}$ and $Y_{\nu, b}$ are the measured data and $X_{\nu, b}^{(n)}$ and $Y_{\nu, b}^{(n)}$ are calculated from the iterated temperature map using Eqs. (3) and (4), $N_{\nu}$ is the number of frequencies and $N_{b}$ is the number of baselines. The squared deviation is weighted by the measurement error $\eta_{\nu, b}^{2}$.

The spatial MEM term is defined, again as in standard MEM, as follows

$S_{\mathrm{spa}}^{(n)}=-\sum_{x, y, \nu} T^{(n)} \ln T^{(n)}, \quad T^{(n)}=T^{(n)}(x, y, \nu)$,

with map temperature, $T$, being a function of spatial position and frequency.

The traditional least-squares MEM maximizes the spatial entropy term, $S_{\text {spa }}^{(n)}$, subject to the data constraints, $\Delta^{(n)}$. In our modified algorithm, we include a spectral entropy term, $S_{\text {spe }}^{(n)}$, which we define in analogy to the spatial term

$S_{\mathrm{spe}}^{(n)}=-\sum_{x, y, \nu} \tau^{(n)} \ln \tau^{(n)}$,

with

$\tau^{(n)}=1+\left|T^{(n)}-T^{\prime(n)}\right|$.

The interpolated temperature, $T^{\prime(n)}$, is a function of spatial position and frequency and is logarithmically interpolated from the temperatures at the two neighboring frequencies $\nu_{+}$and $\nu_{-}$at the same spatial position

$\ln T_{\nu}^{\prime(n)}=\ln T_{\nu_{-}}^{(n)}+\frac{\ln T_{\nu_{+}}^{(n)}-\ln T_{\nu_{-}}^{(n)}}{\ln \nu_{+}-\ln \nu_{-}}\left(\ln \nu-\ln \nu_{-}\right)$.

This can be alternatively written as

$$
\begin{aligned}
& T_{\nu}^{\prime(n)}=\exp \left[\ln T_{\nu_{-}}^{(n)}\left(\frac{\ln \nu_{+}-\ln \nu}{\ln \nu_{+}-\ln \nu_{-}}\right)\right. \\
& \left.+\ln T_{\nu_{+}}^{(n)}\left(\frac{\ln \nu-\ln \nu_{-}}{\ln \nu_{+}-\ln \nu_{-}}\right)\right] \text {, }
\end{aligned}
$$

or further simplified to

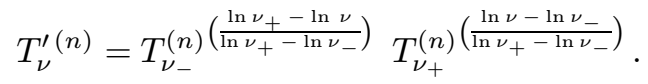

In our implementation, we use Eq. (13) to calculate $T^{\prime}$ and as can be easily seen in Eq. (14) the exponents are only frequency dependent and can be calculated outside the iteration loop. The difference $\left|T^{(n)}-T^{\prime(n)}\right|$ (Eq. (11)) is small if the map is smooth in frequency and large otherwise, thus ensuring spectral smoothness. Since this difference can be exactly zero, we added a constant of unity so that $S_{\text {spe }}$ is zero when $T^{\prime(n)}$ equals $T^{(n)}$.

To maximize the objective function $r^{(n)}$ we have to calculate its derivative with respect to the temperature, $T$, at each spatial position and frequency. This equation is the central part of the algorithm. We like to point out that throughout the rest of this paper, as in the actual algorithm, we use an equivalent formalism and minimize the "negentropy", $-S$, instead of maximizing the entropy.

$$
\frac{\mathrm{d} r^{(n)}}{\mathrm{d} T}=\frac{\mathrm{d} \Delta^{(n)}}{\mathrm{d} T}+\lambda \frac{\mathrm{d} S_{\mathrm{spa}}^{(n)}}{\mathrm{d} T}+\psi \frac{\mathrm{d} S_{\mathrm{spe}}^{(n)}}{\mathrm{d} T} .
$$

The data constraint derivative has the following form

$$
\begin{aligned}
\frac{\mathrm{d} \Delta^{(n)}}{\mathrm{d} T}=2 k & \nu^{2} \delta x \delta y \frac{1}{N_{\nu}} \frac{1}{N_{b}} \sum_{b} \frac{1}{\eta_{\nu, b}^{2}}\left[\left(X_{\nu, b}^{(n)}-X_{\nu, b}\right) \times\right. \\
& {[\cos 2 \pi u x \cos 2 \pi v y-\sin 2 \pi u x \sin 2 \pi v y] } \\
& +\left(Y_{\nu, b}^{(n)}-Y_{\nu, b}\right) \times \\
& {[\cos 2 \pi u x \sin 2 \pi v y+\sin 2 \pi u x \cos 2 \pi v y]] }
\end{aligned}
$$

with $\delta x$ and $\delta y$ being the pixel size in the $x$ - and $y$ direction. For 1 -d data $(v=0)$, the data constraint derivative reduces to

$$
\begin{gathered}
\frac{\mathrm{d} \Delta^{(n)}}{\mathrm{d} T}=2 k \nu^{2} \delta x \frac{1}{N_{\nu}} \frac{1}{N_{b}} \sum_{b} \frac{1}{\eta_{\nu, b}^{2}}\left[\left(X_{\nu, b}^{(n)}-X_{\nu, b}\right) \cos 2 \pi u x\right. \\
\left.+\left(Y_{\nu, b}^{(n)}-Y_{\nu, b}\right) \sin 2 \pi u x\right] .
\end{gathered}
$$

The derivative of the spatial entropy term is simply

$\frac{\mathrm{d} S_{\mathrm{spa}}^{(n)}}{\mathrm{d} T}=1+\ln T^{(n)}$.

The derivative of the spectral entropy term consists of three terms similar to Eq. (18) since $T$ contributes to $\tau(\nu)$, $\tau\left(\nu_{+}\right)$, and $\tau\left(\nu_{-}\right)$. The terms $\frac{\mathrm{d} \tau\left(\nu_{+}\right)}{\mathrm{d} T}$ and $\frac{\mathrm{d} \tau\left(\nu_{-}\right)}{\mathrm{d} T}$ are easily derived from Eq. (14). The superscript $(n)$ is omitted for clarity.

$$
\begin{aligned}
\frac{\mathrm{d} S_{\text {spe }}^{(n)}}{\mathrm{d} T}=(1 & +\ln \tau(\nu)) \frac{\mathrm{d} \tau(\nu)}{\mathrm{d} T}+\left(1+\ln \tau\left(\nu_{+}\right)\right) \frac{\mathrm{d} \tau\left(\nu_{+}\right)}{\mathrm{d} T} \\
& +\left(1+\ln \tau\left(\nu_{-}\right)\right) \frac{\mathrm{d} \tau\left(\nu_{-}\right)}{\mathrm{d} T}
\end{aligned}
$$


with

$$
\begin{gathered}
\frac{\mathrm{d} \tau(\nu)}{\mathrm{d} T}=( \pm 1) \quad \begin{cases}+1, & T>T^{\prime} ; \\
-1, & T<T^{\prime}\end{cases} \\
\frac{\mathrm{d} \tau\left(\nu_{+}\right)}{\mathrm{d} T}=\left(\frac{\ln \nu_{++}-\ln \nu_{+}}{\ln \nu_{++}-\ln \nu}\right) \frac{T^{\prime}\left(\nu_{+}\right)}{T(\nu)}( \pm 1) \\
\begin{cases}+1, & T^{\prime}\left(\nu_{+}\right)>T ; \\
-1, & T^{\prime}\left(\nu_{+}\right)<T\end{cases}
\end{gathered}
$$$$
\frac{\mathrm{d} \tau\left(\nu_{-}\right)}{\mathrm{d} T}=\left(\frac{\ln \nu_{-}-\ln \nu_{--}}{\ln \nu_{-} \ln \nu_{--}}\right) \frac{T^{\prime}\left(\nu_{-}\right)}{T(\nu)}( \pm 1)
$$$$
\begin{cases}+1, & T^{\prime}\left(\nu_{-}\right)>T \\ -1, & T^{\prime}\left(\nu_{-}\right)<T\end{cases}
$$

where $\nu_{++}$is one frequency up from $\nu_{+}$and $\nu_{--}$is one frequency down from $\nu_{-}$. This means that while $T^{\prime}$ is determined from its nearest two neighbors in frequency, $\frac{\mathrm{d} S_{\text {spe }}^{(n)}}{\mathrm{d} T}$ is calculated using the nearest four neighbors. At the ends of the frequency range, the highest and lowest frequencies, $\nu_{N}$ and $\nu_{1}$, have to be treated separately. The ratios of logarithms of frequencies in Eqs. (21) and (22) are about $\frac{1}{2}$ and are easily extrapolated for the highest and the lowest frequencies by assuming that the spacing between frequencies stays the same. At the upper boundary, we substitute the values of $T^{\prime}$ and $\tau$ at $\nu_{N-1}$ for the ones at the frequencies $\nu_{N}$ and $\nu_{N+1}$, and at the lower boundary, we substitute their values at $\nu_{2}$ for the ones at the frequencies $\nu_{1}$ and $\nu_{0}$.

In standard MEM, the choice of Lagrange multiplier $\lambda$ is problematic, and generally must be done with trial and error for a particular application. Our algorithm compounds the problem by introducing an additional parameter $\psi$. We now discuss the choice of these Lagrange multipliers for this application. The reconstruction starts with an initial temperature map, such as a "flat" map or a model of the source, and ends with a final map which is the reconstructed image. The Lagrange multipliers, $\lambda$ and $\psi$, have to balance the three terms of $\frac{\mathrm{d} r}{\mathrm{~d} T}$ in Eq. (15). The spatial entropy term, $\frac{\mathrm{d} S_{\text {spa }}}{\mathrm{d} T}$, is always positive, while the spectral entropy term, $\frac{\mathrm{d} S_{\text {spe }}}{\mathrm{d} T}$, can be either positive, negative or zero. The data term, $\frac{\mathrm{d} \Delta}{\mathrm{d} T}$, is negative if the initial map is a flat map. Thus, the temperature, $T$, will increase if $\frac{\mathrm{d} r}{\mathrm{~d} T}$ is negative and decrease if $\frac{\mathrm{d} r}{\mathrm{~d} T}$ is positive. The initial map is chosen to be smooth everywhere. Therefore, the spectral entropy term will be zero at the first iteration and will remain very small at the first few iterations, and, as a consequence, $\lambda$ can be determined from the initial map and the data constraint.

$\frac{\mathrm{d} r^{(1)}}{\mathrm{d} T}=\frac{\mathrm{d} \Delta^{(1)}}{\mathrm{d} T}+\lambda \frac{\mathrm{d} S_{\text {spa }}^{(1)}}{\mathrm{d} T}$.

The parameter, $\lambda$, has to be small enough to allow the temperature to increase from the initial value at any point in the map, where the source of interest is present, while $\lambda$ should also be large enough to ensure that the temperature stays at the initial value or increases only slightly at other points. If the initial map is a flat map characterized by a single temperature $T_{\min }=T^{(1)}$, this requirement leads to the following relation

$$
\lambda<\frac{1}{1+\ln T_{\min }} \min \left(-\frac{\mathrm{d} \Delta^{(1)}}{\mathrm{d} T}\right)
$$

for all points in the map where the source of interest is present. This relation provides an upper limit for $\lambda$ and since the objective is to reproduce the source and at the same time to reduce ripples due to unmeasured Fourier components, this is the optimum value to use.

For the parameter $\psi$, an analogous relation to Eq. (24) is not useful because most of the iterated maps would have to be taken into account, not just the initial one. To determine the best value of $\psi$, we reconstructed several maps using different values of $\psi$ with the same $\lambda$, derived from Eq. (24), and then compared the spectra of the different maps. As an initial value of $\psi$, we note that the term $\frac{\mathrm{d} S_{\text {spe }}}{\mathrm{d} T}$ is most likely smaller than $\frac{\mathrm{d} S_{\mathrm{spa}}}{\mathrm{d} T}$ since $\tau$ is the difference of two temperatures, thus $\psi=\lambda$ is a good lower limit. For a small $\psi$, the reconstruction will not differ much from a reconstruction using the spatial entropy term alone. With increasing $\psi$, the sidelobes will be steadily reduced, and the spectra will appear steadily smoother, but the overall spectral shape should remain the same. When $\psi$ gets too large, however, the spectral slopes begin to decrease due to overly weighting the spectral smoothness parameter. We have found that this change in spectral character is very pronounced and easily discerned. The optimum value of $\psi$ is then the largest value before which this artificial flattening occurs. We do not yet have enough experience to say whether the optimum value of $\psi$ will be similar for all sources (and spectral shapes), or whether the above procedure must be followed for each individual data set.

\subsection{The 1-d data}

We apply our 1-d algorithm to observations taken with the OVRO frequency-agile interferometer of active region AR 5417 near the solar limb on March 20, 1989 (vernal equinox) using the two 27-m antennas and the 40-m antenna arranged in a linear east-west array with the two $27-\mathrm{m}$ dishes at 122 and $488 \mathrm{~m}$ east and the $40-\mathrm{m}$ dish at $1066 \mathrm{~m}$ east. The array was operated with an observing sequence that sampled 45 frequencies in the range $1-18 \mathrm{GHz}$, in both the right- and left-handed circular polarization every $10 \mathrm{~s}$. The amplitudes and phases were calibrated with respect to our primary flux calibrator, $3 \mathrm{C} 84$, which in turn was calibrated relative to the flux standard 3C 286. Unfortunately, the receiver on the 40-m antenna experienced a hardware problem that resulted in 
no phase lock above $10 \mathrm{GHz}$, and there was external interference at $1.0 \mathrm{GHz}$, so the data presented here range from $1.2-10 \mathrm{GHz}$.

For each of the three baselines, we analyze the observations at the 31 frequencies in the range $1.2-10 \mathrm{GHz}$ taken between 21:05 UT and 23:58 UT. The projected baselines change during this time due to Earth rotation, resulting in fringe spacings that range from $6.8^{\prime \prime}$ at $10 \mathrm{GHz}$ to $276^{\prime \prime}$ at $1.2 \mathrm{GHz}$. By dividing the data into 80 time samples, we get $N_{b}=240$ Fourier points at each observed frequency. To adequately map the active region, we use a map size of $256^{\prime \prime}$ (with a shift of $23^{\prime \prime}$ from the nominal phase center to bring the solar limb to the center of the map) and a spatial resolution or pixel size of $1^{\prime \prime}$. The lowest frequency determines the necessary map size, while the highest frequency determines the required spatial resolution.

Since the active region was located close to the solar limb, we have to correct for the interferometer response to the solar limb. For this purpose, we calculated its response to a uniform disk of the size of the Sun when pointed to the edge of the disk. We used the quiet Sun temperatures measured by Zirin et al. (1991) as the frequency-dependent disk temperature. The resulting amplitudes were generally less than $0.1 \mathrm{sfu}$ (solar flux units) which is within the assumed measurement error (see Eq. (27) below). We subtracted the limb response from the observed data and used the corrected data pairs for the reconstruction.

\subsection{The 1-d implementation}

In this section, we describe the current implementation of our MEM algorithm and give parameter values used to reconstruct an image of active region AR 5417. Different implementations of the MEM algorithm have their advantages and disadvantages, as discussed by Narayan \& Nityananda (1986). After some testing of different schemes, we chose the following method for its speed.

We start with a uniform "flat" map which has the same temperature $T_{\min }$ at all frequencies and spatial positions. For each subsequent iteration $n$, we evaluate the gradient $\frac{\mathrm{d} r^{(n)}}{\mathrm{d} T}$ at each point of the map (Eq. (15)) and according to its sign we either increase or decrease the temperature $T^{(n)}$ by a fraction of its value

$T^{(n+1)}= \begin{cases}T^{(n)}\left(1+\gamma^{(n)}\right), & \frac{\mathrm{d} r^{(n)}}{\mathrm{d} T}<-10^{-6} \\ T^{(n)}\left(1-\gamma^{(n)}\right), & \frac{\mathrm{d} r^{(n)}}{\mathrm{d} T}>10^{-6} \\ T^{(n)}, & \text { otherwise. }\end{cases}$

The gain, $\gamma^{(n)}$, is a function of iteration $n$ only, while $T^{(n)}$ and $\frac{\mathrm{d} r^{(n)}}{\mathrm{d} T}$ are, in addition, functions of spatial position $x$ and frequency $\nu$. We decrease $\gamma$ exponentially with $n$ to make sure that changes in $T$ are getting smaller the closer $T$ gets to the actual temperature value.

$$
\gamma^{(n)}=\exp \left[\left(\frac{\ln \gamma^{\left(n_{\max }\right)}-\ln \gamma^{(1)}}{n_{\max }-1}\right)(n-1)+\ln \gamma_{(1)}\right]
$$

$$
n=1, n_{\max }
$$

where $\gamma^{\left(n_{\max }\right)}, \gamma^{(1)}$, and $n_{\max }$ are parameters to be specified.

The parameters have to be chosen so that any real solar temperature is within the range of reconstructable temperatures; i.e the initial temperature, $T_{\min }$, has to be much smaller than any solar temperature and the largest possible iterated temperature has to be much larger than the largest solar temperature. In addition, the parameters have to be chosen so that the increase at the beginning is not too large in order not to "freeze in" an early iteration; a large $\gamma^{(1)}$ requires a small $T_{\min }$. We set the final gain, $\gamma^{\left(n_{\max }\right)}$, to 0.001 and we use the following additional parameter values of $T_{\min }=100, n_{\max }=250, \gamma^{(1)}=0.5$ to fulfill the constraints.

For the measurement error, $\eta_{\nu, b}$ (in sfu), we use the following relation

$\eta_{\nu, b}=0.05 A_{\nu, b}+0.1$

with $A_{\nu, b}$ being the amplitude.

To estimate the Lagrange multiplier $\lambda$ for the OVRO data of AR 5417, we used Eq. (24), calculated $\frac{\mathrm{d} \Delta^{(1)}}{\mathrm{d} T}$ for the whole map, and from a dirty map reconstruction estimated the position of the source in the map. This led to the following relation at spatial positions where a source is present:

$$
\begin{aligned}
1<\frac{1}{N_{b}} \sum_{b} \frac{1}{\eta_{\nu, b}^{2}}[ & \left(X_{\nu, b}^{(n)}-X_{\nu, b}\right) \cos 2 \pi u x \\
& \left.+\left(Y_{\nu, b}^{(n)}-Y_{\nu, b}\right) \sin 2 \pi u x\right] .
\end{aligned}
$$

The chosen flat map temperature $T_{\min }$ adds the factor $\left(1+\ln T_{\min }\right)^{-1}=0.2$. The estimated value of $\lambda$ is then

$\lambda=0.2\left(\frac{2 k \nu^{2} \delta x}{N_{\nu}}\right)$

With this value of $\lambda$, we calculated several reconstructions using different values of $\psi$. We found that $\psi=\lambda$ was too small to yield improvements beyond those for a reconstruction using the spatial entropy term alone and that $\psi \geq 3 \lambda$ eliminated all ripples but flattened the spectra at high frequencies. Thus, the "best" value of $\psi$ for the analyzed data was chosen to be

$\psi=2 \lambda=0.4\left(\frac{2 k \nu^{2} \delta x}{N_{\nu}}\right)$.

After the MEM reconstruction was done, the reconstructed map was convolved with the so-called "clean" beam, a Gaussian fit to the inner part of the actual beam, to limit the effect of "superresolution" (e.g. Cornwell \& Braun 1989). All imaging algorithms produce some degree of superresolution since they estimate unmeasured Fourier components; i.e. the reconstructed image shows structures 
on spatial scales smaller than the fringe spacing of the largest baseline. A superresolution of a factor two or less is generally acceptable (cf. Narayan \& Nityananda 1986). In our case, we have an additional complication due to the added spectral term which causes the reconstructed map to be spatially not as smooth as it would be without this term, which produces "signal" at high spatial frequencies. We compared spatial power spectra of the convolved restored map with power spectra of the corresponding dirty map and found that the largest spatial frequency present in the reconstructed map is comparable to the one in the dirty map within a factor of 1.5 or less. This implies that convolving with the clean beam sufficiently limits the influence of superresolution and that at the same time its effect is limited enough to preserve the spatial information present in the original data.

In our initial algorithm, used to create the maps in this paper, we directly compute the Fourier transform (Eqs. (5) and (6)) which has the advantage of simplicity over the Fast Fourier Transform (FFT) in that it does not require gridding of the visibilities (see Thompson et al. 1991). However, in looking ahead to the 2 -d version of the algorithm, execution time becomes more important than simplicity. Therefore, we have extended our 1-d algorithm to perform gridding and employ the FFT. Because gridding of multifrequency data introduces special problems, we briefly outline the gridding process here. The gridding process is described mathematically in terms of convolution and resampling, where an appropriate convolution function has to be chosen. A simple choice for a convolution function, and the one we employ, is a rectangular function (cell averaging); the (uv) plane is divided into rectangular cells of dimension $\Delta u$ by $\Delta v$ centered on the grid point and all measurements within each cell are assigned to the grid point.

The problem introduced with gridding of multifrequency data lies in its smoothing effect on the visibilities. For example, if we divide the spatial frequencies between 0 and the Nyquist frequency, $k_{\mathrm{N}}$, into 512 grid points and use a pixel size of $1^{\prime \prime}\left(k_{\mathrm{N}}=\frac{1}{2^{\prime \prime}}\right)$, the $N_{b}=240$ observations occupy 106 grid points at $10 \mathrm{GHz}$ and only 15 at $1.2 \mathrm{GHz}$. To reduce the amount of smoothing at lower frequencies, we divide our frequency range $1.2-10 \mathrm{GHz}$ into three ranges with different spatial resolution and grid each range separately. We choose a low-frequency range $(1.2-2.6 \mathrm{GHz})$ with a pixel size of $4^{\prime \prime}$, a mid-frequency range $(2.8-5.0 \mathrm{GHz})$ with $2^{\prime \prime}$, and a high-frequency range $(5.2-10.0 \mathrm{GHz})$ with $1^{\prime \prime}$ to ensure that the smoothing introduced by the gridding process remains within a factor of two to five. The combined effect of using FFT instead of direct Fourier transform, gridding and dividing into ranges with different spatial resolution reduces the execution time of the MEM algorithm by a factor of $\sim 20$, for the same 1 -d algorithm. The gain is even greater when extended to 2 -d.
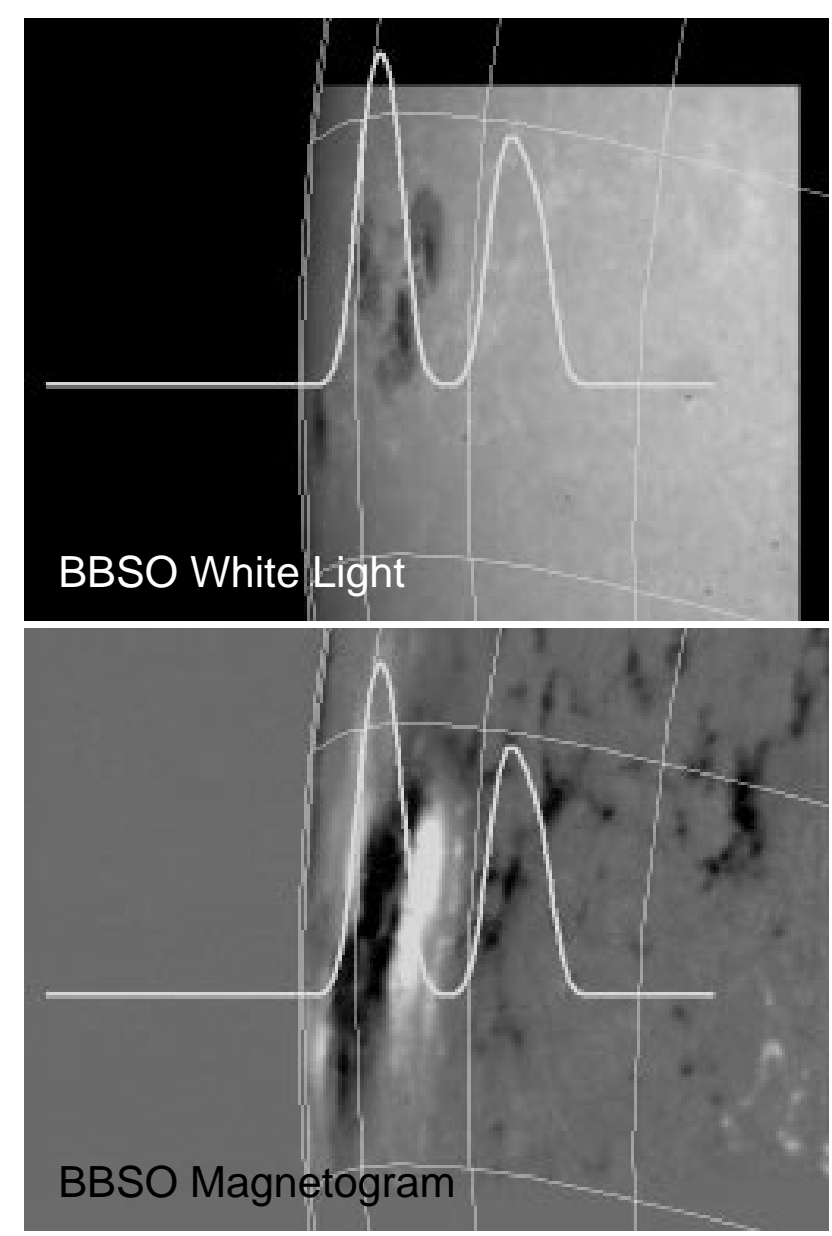

Fig. 1. A white-light image and a magnetogram of active region AR 5417 near the solar limb on March 20, 1989 (vernal equinox) from BBSO. The radio image at $3.6 \mathrm{GHz}$ (covering $256^{\prime \prime}$ ) is superposed for comparison

\section{Application of the algorithm to active region AR 5417}

\subsection{Active region mapping}

Figure 1 shows a white-light image and a magnetogram of AR 5417 taken at Big Bear Solar Observatory (BBSO). The east limb can be seen on the left side of the images, and lines of solar longitude (in steps of $10^{\circ}$ ) and latitude (south $20^{\circ}$ and $30^{\circ}$ ) are shown superposed. The leading sunspot shows positive polarity and the following sunspot shows negative polarity. The active region is surrounded by areas of negative polarity. The radio image at $3.6 \mathrm{GHz}$ covering $256^{\prime \prime}$ (cf. Fig. 4), which was reconstructed from the observations taken between 21:05 UT and 23:58 UT, is superposed for comparison. Note that the radio signal shows two sources, one of them coincides with the active region, while the westward source coincides with the location of an earlier M3.1 flare which erupted at 20:38 UT, about half an hour before our observing time, reached its maximum in soft X-rays at 20:52 UT (about 10 min before 


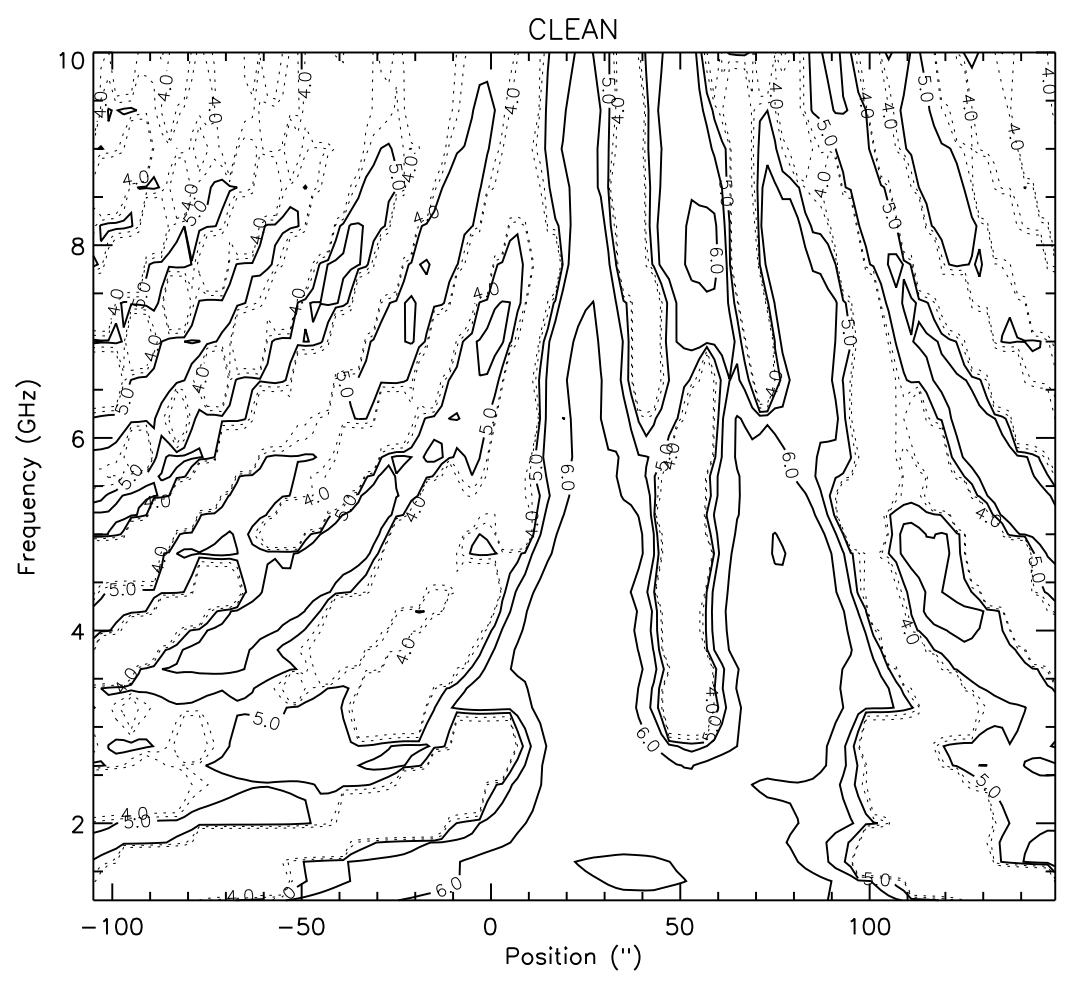

Fig. 2. The CLEAN reconstruction. The spatial information encoded in the spectrum is not used. The contours are at logarithmic intervals to bring out the lower level sidelobes. (contour $4: T_{b}=10^{4} \mathrm{~K}$, contour $5: T_{b}=10^{5} \mathrm{~K}$, contour $6: T_{b}=10^{6} \mathrm{~K}$ )

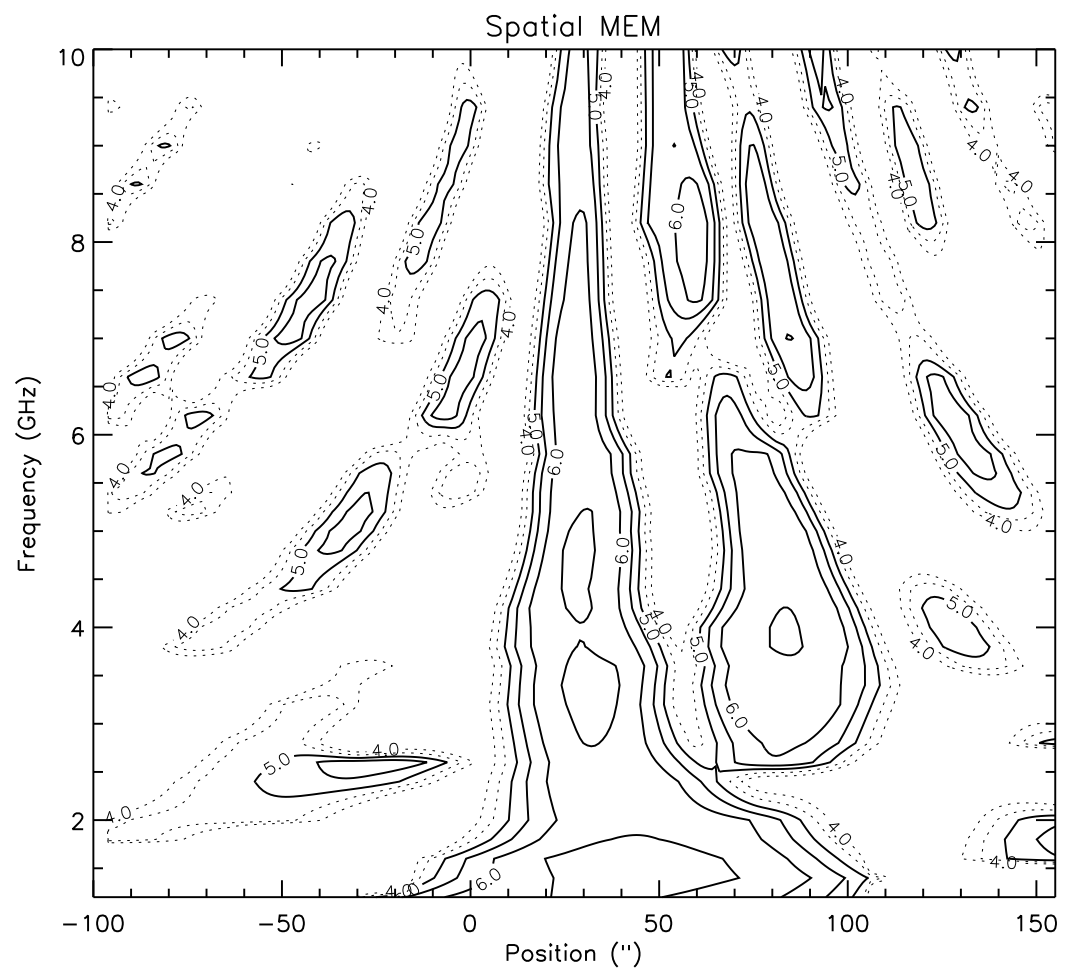

Fig. 3. The spatial MEM reconstruction. The spatial information encoded in the spectrum is not used. The contours are at logarithmic intervals to bring out the lower level sidelobes. (contour $4: T_{b}=10^{4} \mathrm{~K}$, contour $5: T_{b}=10^{5} \mathrm{~K}$, contour $6: T_{b}=10^{6} \mathrm{~K}$ ) 


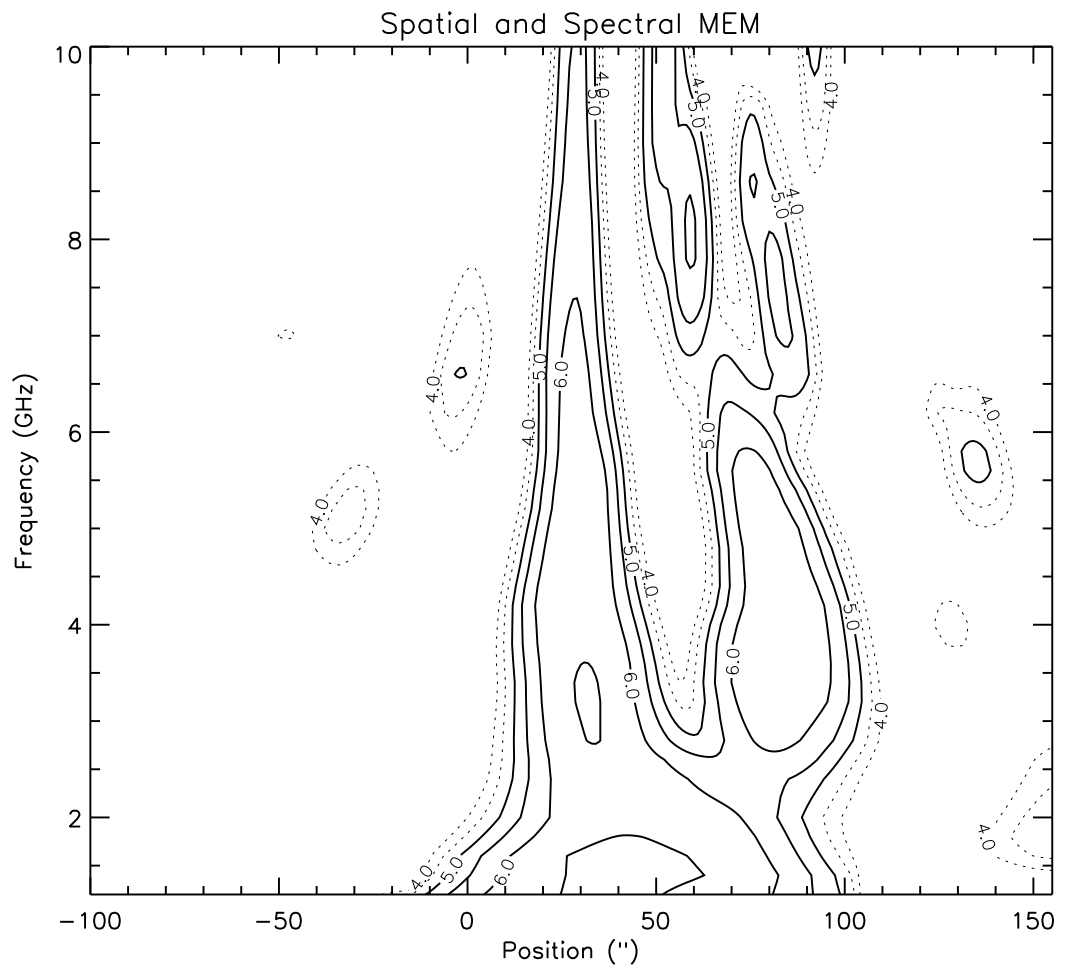

Fig. 4. The reconstructed map using the spatial/spectral MEM algorithm. The contours are at logarithmic intervals to show an improvement in sidelobe level in comparison with Figs. 2 and 3. (contour 4: $T_{b}=10^{4} \mathrm{~K}$, contour 5 : $T_{b}=10^{5} \mathrm{~K}$, contour 6 : $\left.T_{b}=10^{6} \mathrm{~K}\right)$

our observations began), but took several hours to decay. The Big Bear images were taken at 20:01 UT, before the flare occcured.

Figures 2 and 3 show two image reconstructions of the left-hand circular polarization using conventional methods where spectral information is not used. The contours in the figures are in brightness temperature, $T_{b}$, expressed in a logarithmic scale (contour 4: $T_{b}=10^{4} \mathrm{~K}$, contour 5 : $T_{b}=10^{5} \mathrm{~K}$, contour $\left.6: T_{b}=10^{6} \mathrm{~K}\right)$. Figure 2 shows a reconstruction using the CLEAN algorithm, while Fig. 3 shows a reconstruction using only the spatial part of our MEM algorithm $(\psi=0)$. The two reconstructions show a single radio source extending on the spatial axis scale from about $0^{\prime \prime}$ to $+100^{\prime \prime}$ at frequencies below about $2.5 \mathrm{GHz}$. Both show that the active region centered at about $+29^{\prime \prime}$ is visible at all higher frequencies, and they both show the flare-related source to the right of the active region. Other features are sidelobes, which are more pronounced in the corresponding dirty map not shown here. The largest sidelobe temperatures are about one order of magnitude smaller than the temperatures of the active region; both reconstructions suppress sidelobes by about one order of magnitude.

Figure 4 shows the reconstruction of the same radio data using the additional spectral information. The spatial/spectral MEM algorithm reconstruction shows the same sources seen in the two previous figures, but the sidelobes are suppressed by almost two orders of magnitude, which shows that including the spectral information greatly improves the dynamic range. This improvement is best illustrated by directly comparing brightness temperature maps at a given frequency as in Fig. 5 which shows maps at frequencies (a) $2.0 \mathrm{GHz},(b) 5.0 \mathrm{GHz},(c) 7.0 \mathrm{GHz}$, and $(d) 9.0 \mathrm{GHz}$ derived from the spatial/spectral MEM reconstruction (filled gray areas), the spatial MEM reconstruction (solid line) and the CLEAN reconstruction (dashed line). Note that the amplitude scale is logarithmic. Even where the spatial/spectral MEM map shows relatively strong sidelobes, as in Fig. 5b, the sidelobes are about one order of magnitude smaller than in the other two reconstructions.

The reconstructed image of the right-hand circular polarization data is very similar to that in Fig. 4 indicating that the degree of polarization is low. This is to be expected for observations near the limb (cf. Lee et al. 1993), where the magnetic field lines are nearly perpendicular to the line of sight. Spectra from the spatial/spectral MEM reconstruction in both right- and left-hand polarization are used to derive coronal temperatures and magnetic field strengths in the next section.

Now, we briefly discuss the flare-related source located to the west of the active region. In $\mathrm{H} \alpha$ images 

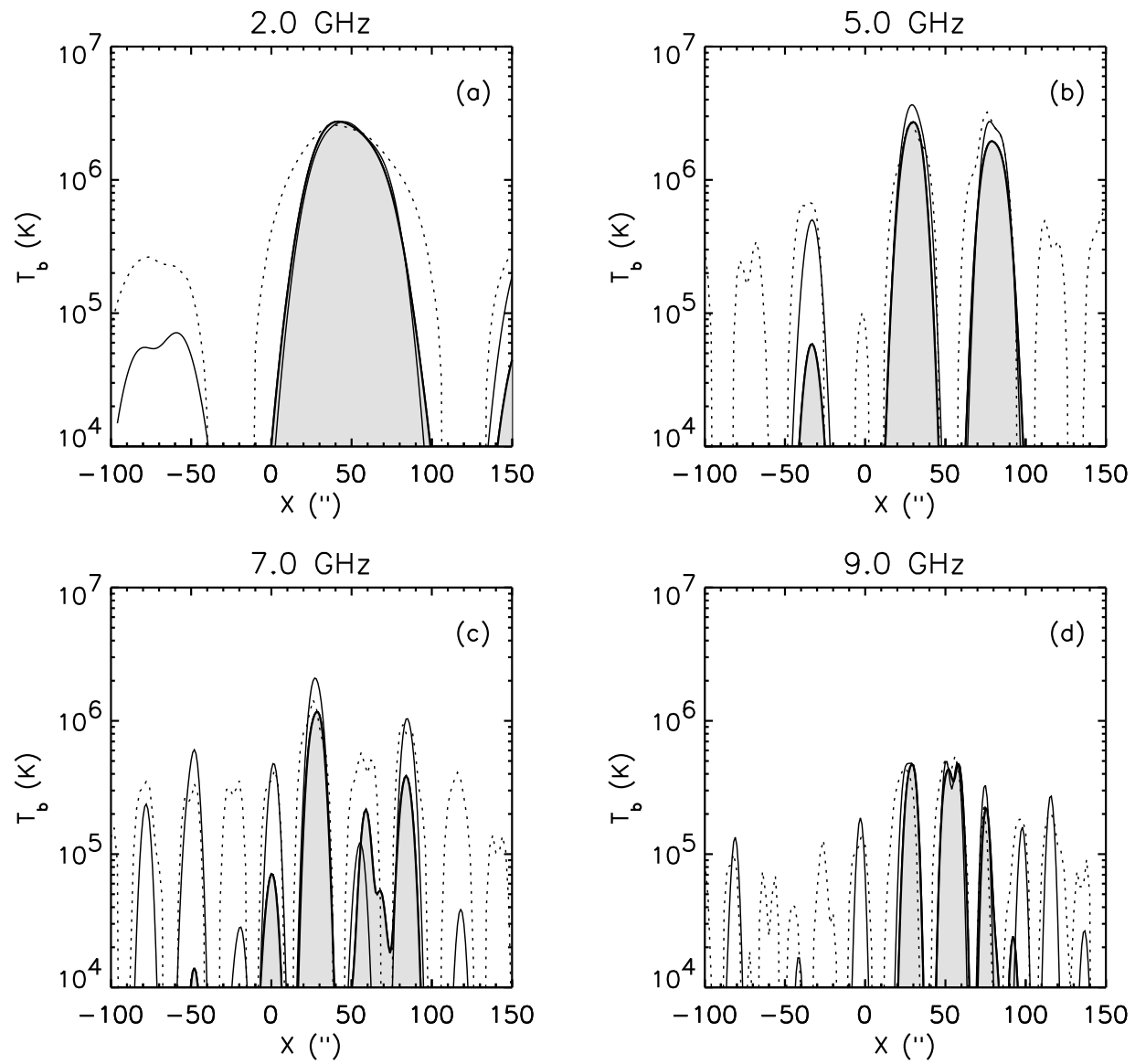

Fig. 5. The brightness temperature maps at frequencies a) $2.0 \mathrm{GHz}$, b) $5.0 \mathrm{GHz}, \mathbf{c}) 7.0 \mathrm{GHz}$, and d) $9.0 \mathrm{GHz}$ of the spatial/spectral MEM reconstruction (filled gray areas), the spatial MEM (solid line) and the CLEAN reconstruction (dotted line)

from BBSO, we found that the flare event comprised two ribbons aligned along the interferometer fringes. Although the nonthermal radio emission was over by the time of our observations, the $\mathrm{H} \alpha$ ribbons remained quite bright and separated slowly in classic post-flare evolution. We know from events seen with Yohkoh that a large, spatially-static soft X-ray loop was likely responsible for the continued radio emission from this location. Due to temporal evolution of this source, however, which can be different at different frequencies, we might expect inconsistencies in the reconstructed image averaged over the entire period as in Fig. 4, where the flare-related source is located at different spatial positions at different frequencies from about $80^{\prime \prime}$ at $3 \mathrm{GHz}$ to about $70^{\prime \prime}$ at $6 \mathrm{GHz}$ and shows two separate sources at higher frequencies centered at about $55^{\prime \prime}$ and $80^{\prime \prime}$. Because the unwanted temporal evolution undoubtedly affects the spectra of this flare-related source, we do not attempt to interpret it here. Instead, we focus on the active-regionrelated source. To see to what extent the flare-related source influences the active-region-related source, we divided the data set into six subsets of equal length of about $\frac{1}{2}$ hour duration and reconstructed maps from these subsets. Although the reconstructions were based on fewer visibilities, we found that the active-region-related source gave a consistently reconstructed morphology in all subsets except the first. In this first subset, an apparent splitting of the active-region source above $6 \mathrm{GHz}$ was seen, likely an inaccurate reconstruction due to temporal variations in the shape of the flare source during this first half-hour period. In the other five time periods, the shape of the flare-related source was quite stable while its brightness decreased slowly. The ratio of flare to active region maximum temperature, averaged over $2.6-10.0 \mathrm{GHz}$, is 1.6 in the first period, decreases to 1.2 in the second, 0.8 in the third and remains 0.7 in the last three subsets. Thus, the emission of the active-region-related source integrated over the entire period should be only slightly affected by the flare-related source. We assume that the shape of the active-region-related source is well represented in the synthesis map of Fig. 4. In any case, our main purpose here is to show the relative improvement of the MEM algorithm over other reconstruction methods. 


\subsection{Active region spectra}

Figure 6 shows two examples of brightness temperature spectra derived from the spatial/spectral MEM reconstruction $(\square)$, the spatial MEM reconstruction $(\times)$, and the CLEAN reconstruction $(+)$. Some assumptions are necessary for deducing the brightness temperature from observations with one-dimensional spatial resolution. At each iteration of the algorithm, the model brightness temperature map must be converted to solar flux units to compare with the measured amplitudes. This is done assuming an area for each pixel of $1^{\prime \prime}$ square. Thus, the flux in each pixel of the 1-d model is imagined to be condensed into a single $1^{\prime \prime}$ pixel along the fringe direction. The algorithm thus produces a map whose brightness is too high by the amount of the extent of the source along the fringes, in arcseconds. Of course, the true source extent is unknown, but we make the assumption that the sources are the same size along the fringes as they are across the fringes (that is, they are circular in shape), and have corrected the brightness temperatures accordingly. This modification was made for Figs. 3 and 4, and also in the spectra that are discussed in this section.

At position $+33^{\prime \prime}$ (Fig. 6a), which corresponds to the center of the active region source, the spectra stay more or less flat below about $5 \mathrm{GHz}$ and decrease at higher frequencies with a steep slope of -5 , which indicates that the emission is optically thick below $5 \mathrm{GHz}$ and that thermal gyroresonance is the emission mechanism (e.g. Gary $\&$ Hurford 1994). For an isothermal corona, the optically thick part of the spectrum should be perfectly flat. The appearance of apparently real deviations from a flat spectrum in Fig. 6 could be due to real variations of temperature with height in the active region, or could be artificial due to our assumption of a circularly shaped source at all frequencies.

The spatial MEM leads generally to slightly higher temperatures than the spatial/spectral MEM, while CLEAN produces smaller temperatures. This may be due to a redistribution of the flux into sidelobes in the latter case. The transition from optically thick to optically thin occurs at almost the same frequency and the highfrequency slope is also more or less the same for the three different reconstructions. The spatial/spectral MEM reconstruction shows the smoothest spectrum of the three due to the added frequency constraint, but all three methods do a reasonable job for this position of the strongest source in the maps.

At position $+39^{\prime \prime}$ (Fig. 6b), which is farther from the center of the active region, the spectra are flat for frequencies below $3.4 \mathrm{GHz}$ and the high-frequency slope is -4 which is still too steep for free-free emission, and so is likely to be produced by gyroresonance. Again, the temperatures of the spatial MEM reconstruction are, in general, slightly higher than the ones of the spatial/spectral MEM resconstruction, while the CLEAN reconstruction
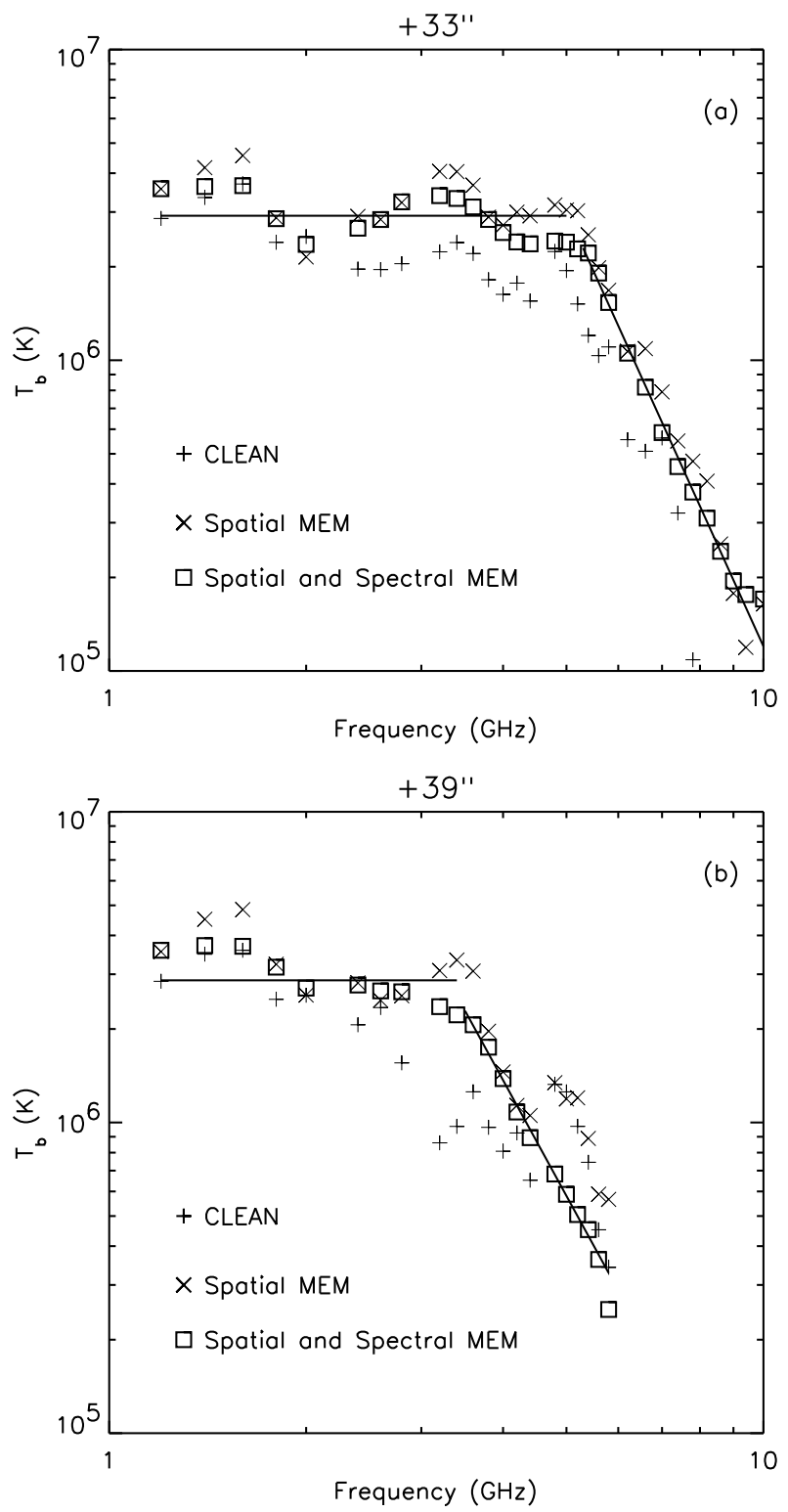

Fig. 6. The brightness temperature spectra at spatial positions a) $+33^{\prime \prime}$ and b) $+39^{\prime \prime}$. Each plot shows spectra derived from the spatial/spectral MEM reconstruction ( $\square$ ), the spatial MEM reconstruction $(\times)$, and the CLEAN reconstruction $(+)$

produces smaller temperatures. The high-frequency slope is easily determined in the spatial/spectral MEM reconstruction, while it is rather difficult in the other two reconstructions. This clearly shows the advantage gained by including the spectral smoothness criterion.

The transition between the optically thick and optically thin parts of the spectrum is a sharp "kink" in the spatial MEM reconstruction, while it rolls off more smoothly in the spatial/spectral MEM reconstruction. The smooth transition is perhaps an undesired effect of using the spectral smoothness criterion, so one must 
recognize the potential for artificially smoothing sharp variations in the spectrum. For larger $\psi$, this becomes more pronounced and can, in extreme cases, significantly alter the spectrum. As discussed in Sect. 2.1, we used this behavior to determine a value of $\psi$ that minimizes the unwanted smoothing.

The spectra can be interpreted using knowledge about radio emission mechanisms such as thermal bremsstrahlung (free-free) emission and thermal gyroresonance present in the solar corona (e.g., Dulk 1985; Gary \& Hurford 1994). The average brightness temperature of the flat part of a thermal spectrum gives directly the electron temperature, $T_{\mathrm{e}}$, independent of the emission mechanism. Figure 7 shows the electron temperature, $T_{\mathrm{e}}$, derived from the spectra as a function of spatial position. The solid line represents $T_{\mathrm{e}}$ of the active region centered at about $+29^{\prime \prime}$, while the dotted line gives $T_{\mathrm{e}}$ of the low-frequencies (below $2 \mathrm{GHz}$ ) source. The data are smoothed by a $5^{\prime \prime}$ running mean. The temperature of the active region is about $310^{6} \mathrm{~K}$ with a slight maximum at $+37^{\prime \prime}$, while the temperature of the broad single source is everywhere somewhat higher with a broad maximum at $+38^{\prime \prime}$. At spatial positions $>80^{\prime \prime}$ and at positions $<14^{\prime \prime}$, the emission is optically thin, the presented values are thus lower limits to the true temperature.

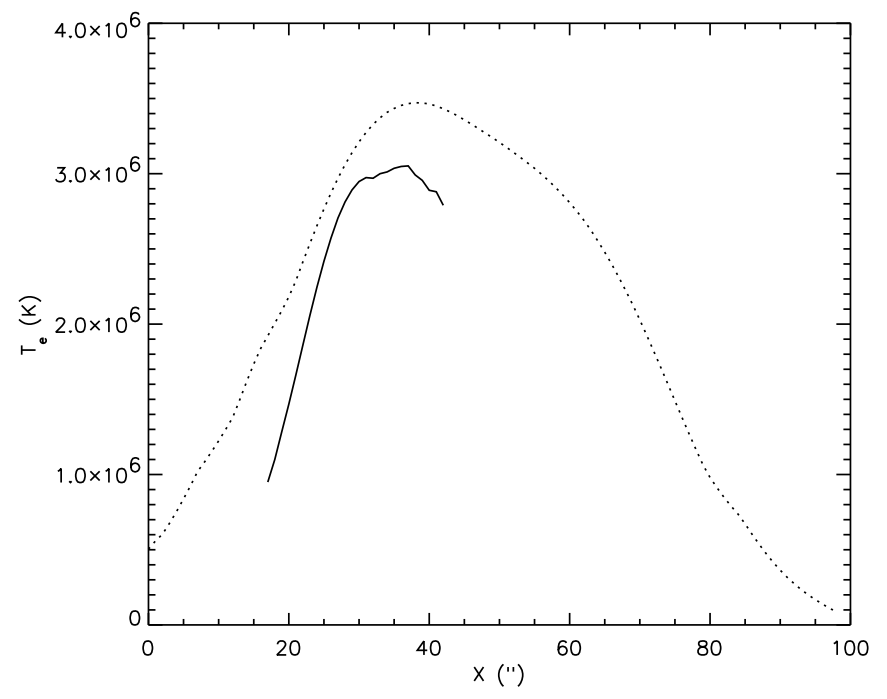

Fig. 7. The electron temperature measured from the flat parts of the brightness temperature spectra $\left(\nu<\nu_{\tau=1}\right.$ : solid line, $\nu<2$ GHz: dotted line)

From the shape of the spectra, we have already noted that thermal gyroresonance is the dominant emission mechanism throughout the active region. This allows us to derive the magnetic field strength in the solar corona

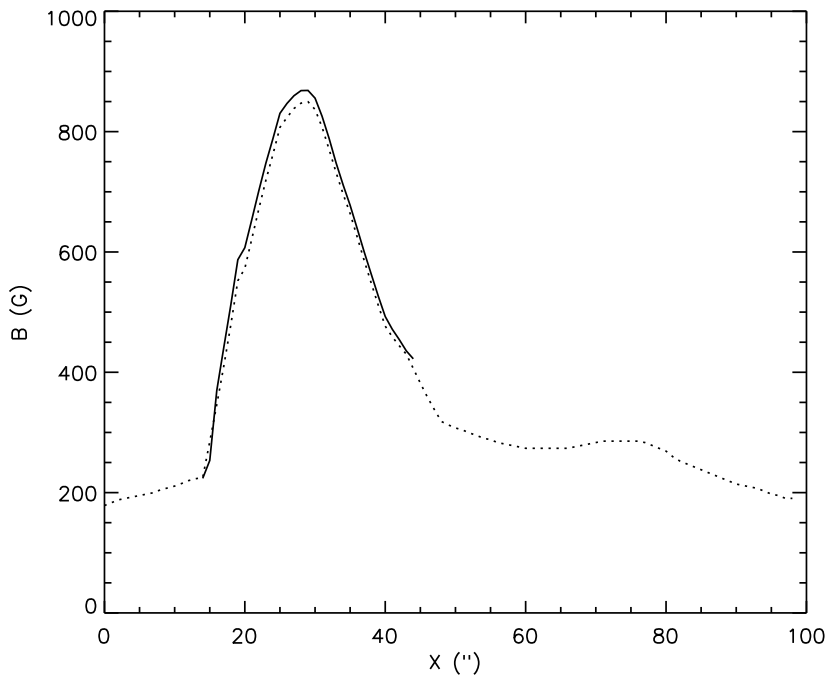

Fig. 8. The total magnetic field strength at the base of the corona assuming $s=3$ everywhere measured from the gyroresonance spectra $\left(\nu<\nu_{\tau=1}\right.$ : solid line, $\nu<2 \mathrm{GHz}$ : dotted line)

following the procedure described by Gary \& Hudford (1994). The highest magnetic field strength is given by

$B_{\max }=\frac{\nu_{\tau=1}}{2.810^{6} \mathrm{~s}}$

where $s$ is the harmonic number. The frequency at which the optical depth is unity $\left(\nu_{\tau=1}\right)$ is just above the frequency where the spectrum turns over, so we determine $\nu_{\tau=1}$ at $\frac{1}{\mathrm{e}} T_{\mathrm{e}}$. Since $\nu_{\tau=1}$ is continous with spatial position, we assume $s=3$ at all positions following Gary \& Hurford (1994) and Lee et al. (1993). Figure 8 shows the total magnetic field strength at the base of the corona. The derived value depends slightly on the value of $T_{\mathrm{e}}$, so we plot two curves. The solid line shows the magnetic field strength derived with $T_{\mathrm{e}}$ given by the solid line curve in Fig. 7, while the dotted line uses $T_{\mathrm{e}}$ from the dotted line in Fig. 7. Both temperatures give the same result, that the magnetic field strength of the active region is about $800 \mathrm{G}$ with a maximum of $870 \mathrm{G}$ at spatial position $+28^{\prime \prime}$ (which is $9^{\prime \prime}$ to the east of the position of maximum temperature).

\section{Discussion and conclusion}

In this work, we introduce a MEM algorithm that produces a global solution to the visibilities in both the spatial and spectral domains and we apply its 1-d implementation to observations of AR 5417 taken with the OVRO frequency-agile interferometer on March 20, 1989 (vernal equinox). Our results show that including the spectral MEM term greatly improves the dynamic range of the reconstructed image (Fig. 4) compared with a reconstruction using the CLEAN algorithm (Fig. 2) or a reconstruction using the spatial MEM term only (Fig. 3). Thus, the 
derived spectra (Fig. 6) show less confusion due to better suppression of sidelobes and, as a direct consequence, physical quantities such as the magnetic field strength can be determined more reliably.

So far, we have stressed the differences between the different reconstructions to show the strength of our method. The similarities can be used to discuss the reliability of the reconstructed image which is a non-trivial problem common to all imaging algorithms (cf. Christiansen \& Hogbom 1985). An imaging algorithm selects among numerous alternative reconstructions which are compatible with the data by using assumptions about the radio source as substitutes for unmeasured Fourier components. Thus, the reconstructed image is an approximation of the real image and depends to a certain degree on the underlying assumptions and on the algorithm used. To address this problem, we repeat the reconstruction with different algorithms. The results (Figs. 2-4) show that our spatial/spectral MEM algorithm is indeed stable and reliable.

The reconstructed brightness temperature spectra in Fig. 6 are spectra typical for active regions with thermal gyroresonance as the dominant emission mechanism. We determine the electron temperature in the region to be $T_{\mathrm{e}} \sim 310^{6} \mathrm{~K}$ (Fig. 7) which is higher than temperatures of $1-210^{6} \mathrm{~K}$ as reported by Gary \& Hurford (1994) and Lee et al. (1993). However, all three different reconstructions in Fig. 6 show similarly high temperatures, so these values are not artifacts of any particular reconstruction algorithm. Therefore, either (1) the electron temperature was indeed $310^{6} \mathrm{~K}$ in AR 5417, or (2) we underestimate the true size of the active region, which is used to scale the temperatures and which we assumed to be the same along and across the fringes, or (3) the elevated temperatures are due to the effects of the flare-related emission. The information in the data set does not allow us to discriminate among these explanations.

Using the turnover, or break, in the gyroresonance spectra, we determine the magnetic field strength of the active region. We find a maximum field strength of $B_{\max } \approx 870 \mathrm{G}$ (Fig. 8), which is low relative to measurements near the center of the disk (Gary et al. 1993), but may compare favorably with measurements near the limb (Lee et al. 1993; Gary \& Hurford 1994). We find that the peak of the magnetic field strength does not coincide with the peak of the electron temperature which agrees with observations by Gary \& Hurford (1994) and might be attributed to the fact that microwave emission appears to be brightest over sunspot penumbrae rather than their umbrae (e.g., White et al. 1992).

We conclude that the spatial/spectral MEM algorithm can greatly improve image reconstruction in applications where the spatial information at different frequencies or energies is not identical, and can be assumed to vary slowly with frequency. Future plans include development of the algorithm for two spatial and one spectral dimensions for application to data taken with the five-element OVRO Solar Array. We anticipate that this algorithm will improve the analysis of our data not only due to the improvement in dynamic range but also by speeding up the analysis and making it less subjective (as compared to CLEAN).

Acknowledgements. We thank Jay Bromley who did the initial programming as an undergraduate student at Caltech. This work was supported by NSF grants ATM-9311416 and AST-9314929, and NASA grant NAGW-4563 to the California Institute of Technology.

\section{References}

Bevensee R.M., 1993, Entropy Solutions to Scientific Problems, Prentice Hall

Christiansen W.N., Hogbom J.A., 1985, Radio telescopes, Cambridge University Press, Cambridge, p. 207

Cornwell T., Braun R., 1989, in Synthesis Imaging in Radio Astronomy, Perley R.A., Schwab F.R. and Bridle A.H. (eds.), ASP Conf. Ser. 6, 167

Dulk G.A., 1985, ARA\&A 23, 169

Gary D.E., Hurford G.J., 1994, ApJ 420, 903

Gary D.E., Leblanc Y., Dulk G.A. Golub L., 1993, ApJ 412, 421

Högbom J., 1974, A\&AS 15, 417

Lee J.W., Gary D.E., Hurford G.J., 1993, Solar Phys. 144, 45

Lee J.W., Hurford G.J., Gary D.E., 1993, Solar Phys. 144, 349

Narayan R., Nityananda R., 1986, ARA\&A 24, 12

Thompson A.R., Moran J.M., Swenson Jr. G.W., 1991, Interferometry and Synthesis in Radio Astronomy. Krieger Publishing Company

White S.M., Kundu M.R., Gopalswamy N., 1992, ApJS 78, 599

Zirin H., Baumert B.M., Hurford G.J., 1991, ApJ 370, 779 